\title{
ANT2 mediates hypoxia and inflammation in obesity
}

Obesity is known to induce adipose tissue hypoxia, leading to an increase in levels of adipocyte hypoxiainducible factor $1 \alpha$ (HIF1 $\alpha)$. This increase in HIF1a is proposed to trigger adipose tissue inflammation and insulin resistance. New research suggests that the mitochondrial protein adenine nucleotide translocase 2 (ANT2) might have a role in this sequence of events.

Previously, increased free fatty acid levels have been shown to stimulate an ANT2-dependent increase in uncoupled mitochondrial respiration. "In the present study, we set out to test whether adipocyte ANT2 is necessary for obesity-induced increased adipocyte oxygen consumption and relative hypoxia," say Yun Sok Lee and Jerrold Olefsky, corresponding authors.

To address their hypothesis, the researchers generated adipocytespecific ANT2-knockout (ANT2 AKO) mice using the Cre-loxP system.

Changes in adipocyte mitochondrial number, mass and activity were then assessed, along with adipocyte oxygen demand and HIF1a expression levels. Knockdown of ANT2 was found to reduce adipocyte oxygen consumption and improve hypoxia (without effects on mitochondrial number or mass). In turn, HIF1a expression levels were reduced.

Macrophage infiltration and proliferation in adipose tissue was measured, along with adipokine expression levels, showing that ANT2 AKO mice were protected from adipose inflammation and fibrosis. ANT2 AKO mice also had improved glucose tolerance and insulin sensitivity.

The role of ANT2 in established glucose intolerance and insulin resistance was also investigated using an inducible ANT2 AKO mouse strain. "[We] showed that ANT2

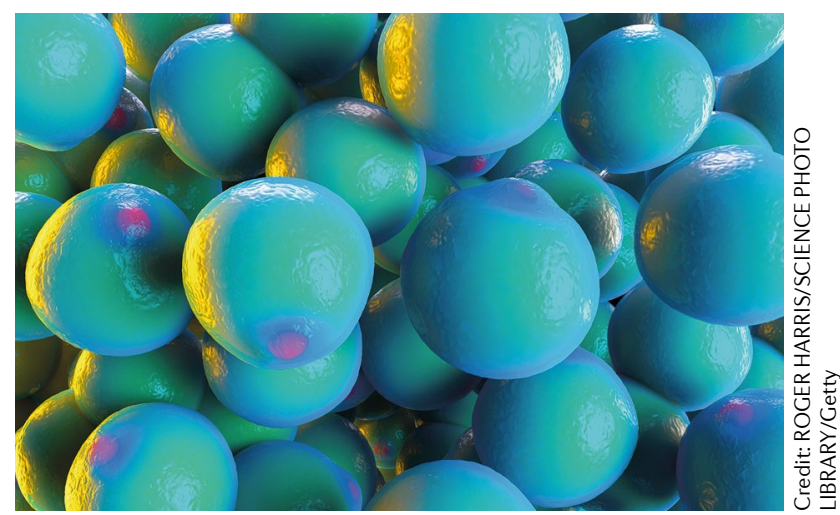

activation is important not only for the initiation of obesity-induced adipose tissue hypoxia, but also for the maintenance of obesity-induced metabolic dysfunction," say Lee and Olefsky.

The researchers conclude that ANT2 warrants further investigation as a target for antidiabetic therapies. Furthermore, several features of high-fat diet fed ANT2 AKO mice resemble those of metabolically healthy obese individuals, raising the possibility that adipocyte ANT2 activity might be associated with metabolic health (or lack thereof) in obesity.

Isobel Leake antidiabetic therapies
ORIGINAL ARTICLE Seo, J. B. et al. Knockdown of ANT2 reduces adipocyte hypoxia and improves insulin resistance in obesity. Nat. Metab. https://doi.org/10.1038/s42255-018-0003-x (2018)

\section{DIABETES}

\section{Skin autofluorescence predicts T2DM}

In order to reduce the burden of type 2 diabetes mellitus (T2DM) on the health of patients, early risk assessments are required. An effective non-invasive technique to assess a patient's risk, however, has been lacking. Previous studies have shown that high skin autofluorescence is indicative of cardiovascular complications and mortality related to T2DM, but a comprehensive analysis to establish whether this measurement could be used as a predictive biomarker in the clinic had not been conducted, until now. New research shows that a non-invasive skin autofluorescence measurement can predict T2DM and cardiovascular disease risk.

The autofluorescence measurement is based on the detection of advanced glycation end products (AGEs) in the skin. AGEs are a heterogeneous group of complex molecules that form non-enzymatically in multistep reactions between reducing sugars and proteins, lipids or nucleic acids.
AGEs accumulate in skin and the concentration of AGEs in the skin can be assessed easily using an AGE reader, which measures skin autofluorescence.

"I introduced this measurement when screening participants at the beginning of the LifeLines Cohort Study in 2006," explains Bruce Wolffenbuttel, corresponding author on the study. "This is the first study on the predictive power of skin autofluorescence in the general population with this number of participants (>70,000)."

The authors recorded baseline skin autofluorescence at the start of the study. Four years later they analysed how well this measurement could predict whether patients developed T2DM and/or cardiovascular disease. They found that, compared with participants who lived disease free, baseline skin autofluorescence was increased in patients who developed T2DM or cardiovascular disease, or who died. Notably, skin autofluorescence worked as a predictive tool independent

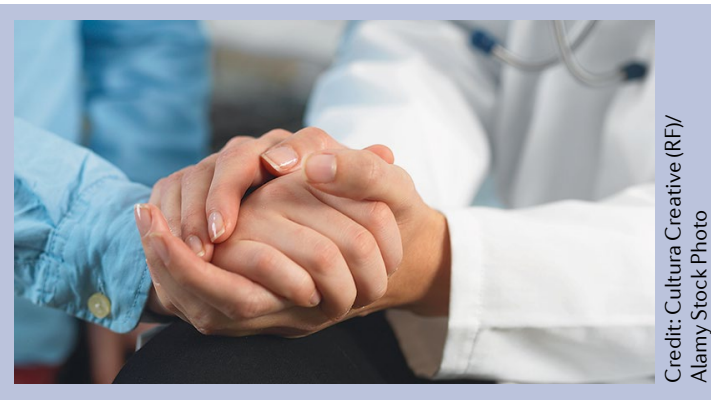

of other risk factors, such as age, sex, waist circumference or smoking status. Wolffenbuttel hopes that this method will be widely used in the future. "We are currently calculating how we can improve the ASCVD and/or Framingham risk score with this measurement,"
We are currently calculating how we can improve the ASCVD and/or Framingham risk score... concludes Wolffenbuttel. We would also like to investigate how the device can be used as pre-screening before doing oral glucose tolerance tests and other diagnostic work-up for possible T2DM."

Anna Kriebs, Associate Editor, Nature Communications

ORIGINAL ARTICLE van Waateringe, R. P. et al. Skin autofluorescence predicts incident type 2 diabetes, cardiovascular disease and mortality in the general population. Diabetologia https:// doi.org/10.1007/s00125-018-4769-x (2018) 